\title{
Performance Evaluation of Spectrum Detection in Cognitive Radio Network
}

\author{
Shubhangi Mahamuni, Vivekanand Mishra \\ Department of Electronics, SVNIT, Surat, India \\ Email: shubhangim11@gmail.com, vive2009@gmail.com
}

Received 16 September 2014; revised 28 October 2014; accepted 18 November 2014

Copyright (C) 2014 by authors and Scientific Research Publishing Inc.

This work is licensed under the Creative Commons Attribution International License (CC BY). http://creativecommons.org/licenses/by/4.0/

(c) (i) Open Access

\begin{abstract}
In mobility management, detection of spectrum hole plays very important role for doing spectrum handoff mechanism. Operation of the cognitive radio depends on the spectrum sensing which is the most important task in case of cognitive radio network. Detection of spectrum holes (underutilized sub-bands of the radio spectrum) estimates average power, throughput and utilized time for each spectrum hole. But in case of reactive spectrum handoff mechanism, spectrum handoff delay becomes tedious task. So in order to make spectrum handoff mechanism efficient our scheme proposed spectrum handoff mechanism with negligible spectrum handoff delay. In this paper we had real time experimental setup with the spectrum handoff mechanism. We investigate algorithm for spectrum handoff mechanism which strongly minimizes spectrum handoff delay of cognitive radio network using spectrum detection method for sensing spectrum holes.
\end{abstract}

\section{Keywords}

Cognitive Radio, Spectrum Sensing, Spectrum Handoff, Spectrum Handoff Minimization

\section{Introduction}

Nowadays, poor utilization of licensed spectrum creates spectrum scarcity. For improved spectrum utilization [1], cognitive radio, a new technology introduced in wireless communications, has a solution to solve the problem of spectrum underutilization [2] [3]. Certain frequency band can only be occupied by the specific licensed users according to FCC's regulation for the radio spectrum, who have full authority on the spectrum access to this frequency band [4]. In cognitive radio the spectrum sensing plays important role. Spectrum sensing and availability of spectrum should do by cognitive radio. If the licensed user uses spectrum, cognitive radio should keep silent [5]; otherwise, cognitive radio can access this spectrum. The detail information of minimization of the interference [6] to the primary radio while meeting the requirement of opportunistic spectrum utilization was 
obtained. Development of efficient algorithms to solve for the optimal solutions as well as computational complexity is reduced and obtained solutions for more general cases. The performance of spectrum sensing based on energy detections is given in [7]. Here an estimated noise variance is used to calculate the threshold used in the spectrum sensing based on energy detection. They proposed a new analytical model to evaluate the statistical performance of the energy detection with cooperative spectrum sensing method. The sensing-throughput tradeoff problem studied [8] and energy detection sensing scheme used to prove cooperative sensing using multiple mini-slots or multiple secondary users. In [9] a survey of spectrum sensing methodologies for cognitive radio is presented. Challenges associated with spectrum sensing were given and enabling spectrum sensing methods were reviewed. The cooperative sensing concept and its various forms are discussed. External sensing algorithms and other alternative sensing methods are discussed. Moreover, statistical modeling of network traffic and utilization of these models for prediction of primary user behavior was studied. The performances and complicated scenarios of the latest spectrum sensing schemes were analytically compared [10]. A macroscopic view of spectrum sensing has been given. Here uncertainty and inter-CRN interference were considered. A scheme called weighted sequential probability ratio test (WSPRT) discussed in detail [11]. Detection of advanced television systems, digital television and generic land mobile radio signals [12] [13] and spectrum sensing and spectrum reconstruction under the umbrella of cognitive radio which is the smart radio to explore and exploit the free spectrum is studied. Here spectrum analyzer was used to emulate cognitive radio to do spectrum sensing. Quick and semi-continuous measurements have been carried out and the information derived about the spectrum under investigation is used for further investigations. Total variation (TV) reconstruction method [14] was employed to rebuild the spectrum and find the boundary of the frequency band which was occupied. In [15] spectrum aware mobility management scheme is explained taken into consideration for the design of our model which utilizes target cell and spectrum pool for doing spectrum handoff mechanism. In case of performance of cognitive radio spectrum access with intra- and inter-handoff [16] is considered for the determination of the rate of inter-handoff. In modeling and analysis of reactive decision spectrum handoff in cognitive radio networks [17] development of Markov transition model integrating with M/G/1 queuing network is used to characterize multiple handoff delay resulting from the sensing time and the waiting time. The detail study of latency requirement for the design of admission control of secondary users was carried out under various traffic arrival rates and service time [18]. A novel geographical fingerprint scheme has been designed to improve its performance during handoff mechanism [19]. Most of the challenges occurred in spectrum handoff mechanism are listed in [20] [21]. The method of retransmission of the collided packets using discrete-time Markov chain has been used in the performance analysis of spectrum handoff for cognitive Radio ad-hoc networks without common control channel under homogeneous traffic [22]. In an optimal spectrum handoff scheme which is designed for the cognitive mobile ad-hoc networks developed co-operative spectrum searching approach is designed. Also a geographical approach is utilized to deal with space handoff. In our proposed model, experimental setup consisting of spectrum analyzer, antenna, lab-view is used for spectrum sensing. This spectrum sensed is utilized for the decision of spectrum handoff. By using prior spectrum sensing information, the information is broadcasted to the nearby users which minimizes the delay that occurred during the spectrum handoff mechanism.

\section{Spectrum Sensing}

\subsection{Sensing Setup}

A computer/laptop, spectrum analyzer and one omni-directional antenna forms practical setup for spectrum sensing as shown in Figure 1. For real time sensing the spectrum and for analysis of the same spectrum analyzer is used. A national instrument (NI) LabVIEW-8.5 is used for spectrum analysis. GPIB cable is connected between the computer and the spectrum analyzer. For all sensing scenarios a linearly polarized omni-directional antenna with $50 \mathrm{ohm}$ unbalanced feed impedance is used. Figure 1 shows the setup diagram.

Most important real time and complete utilization of frequency spectrum [1]-[3] is the required data for the cognitive radio communication. For real time sensing our setup is the device based setup which is useful for spectrum sensing and recording of spectrum signals on spectrum analyzer automatically. This information would be required for spectrum signal analysis in cognitive radio communication. For the continuous measurements of idle time and busy time of licensed users the time which we used for the formation of algorithm for doing spectrum handoff. Further which we have been used for the spectrum handoff delay minimization in sec- 
tion III which includes the sweep time and time to record/save data.

\subsection{Sensing Scenario}

Four sensing scenarios are considered in this paper. The four spectrums are considered for sensing CDMA signal, GSM signal, Wi-Fi, cable TV.

Following is the description of four scenarios as considered here [4] [5]:

a) When a call connection is made from CDMA based cell phone to land-line based phone, the spectrum of CDMA signal is sensed. The sensed frequency band is from $800 \mathrm{MHz}$ to $1100 \mathrm{MHz}$.

b) When a call connection is made from GSM based cell phone to land-line based phone, the spectrum of GSM signal is sensed. The sensed frequency band is from $1700 \mathrm{MHz}$ to $2000 \mathrm{MHz}$.

c) When a Wi-Fi connection is established on notebook computer, the spectrum of Wi-Fi signal is sensed. The sensed frequency band is from $2300 \mathrm{MHz}$ to $2600 \mathrm{MHz}$.

d) A DTV signal is sensed from $697.5 \mathrm{MHz}$ to $704.5 \mathrm{MHz}$.

\subsection{Sensing Result}

Four sensing scenarios are considered in this paper. The four spectrums are considered for sensing CDMA signal, GSM signal, Wi-Fi, cable TV as shown in Figure 2.

Following parameters are used in all sensing scenarios:

- Resolution bandwidth $=20 \mathrm{kHz}$.

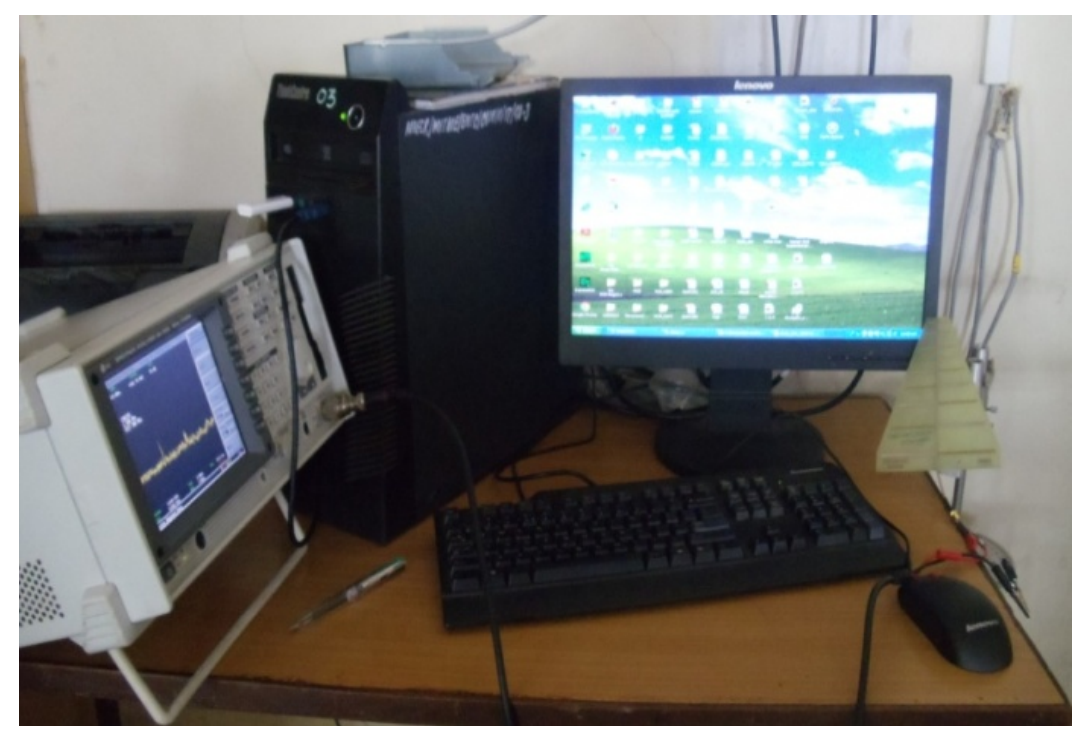

Figure 1. Practical setup.

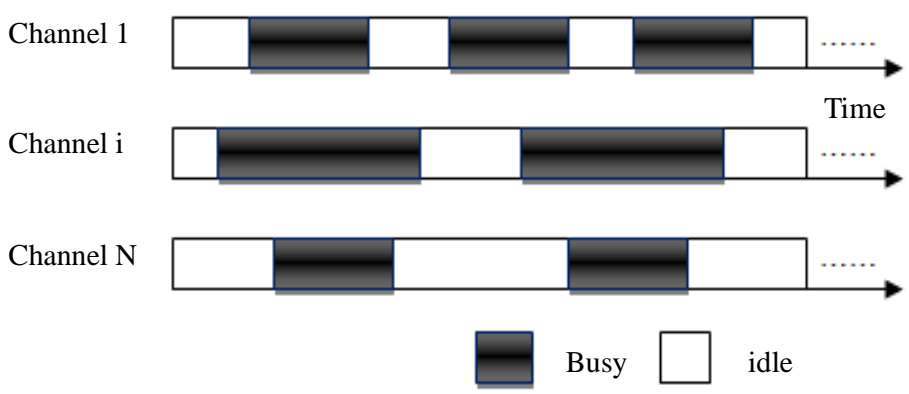

Figure 2. PU activity model. 
- Video bandwidth $=20 \mathrm{kHz}$.

- Sweep time $=15 \mathrm{msec}$.

- $\mathrm{RF}$ attenuation $=10 \mathrm{~dB}$.

- Trace type $=$ clear $/$ write.

- Sweep mode = single sweep display ON.

- Number of points $=700$ points per sweep.

Figure 3 shows spectrum of CDMA signal. In Figure 4, the strong peaks between $800 \mathrm{MHz}$ and $900 \mathrm{MHz}$ show the CDMA signal, when a call connection is established between a CDMA based cell phone and a landline based phone [6]. Whereas, the blank spaces between these strong peaks correspond to the time when call connection is not established.

Figure 4 shows 3-D spectrum of GSM signal. In Figure 5, strong peaks between $1850 \mathrm{MHz}$ and $1950 \mathrm{MHz}$ show the GSM signal, when a call connection is established between a GSM based cell phone and a land-line based phone [7] [8]. Whereas, the blank spaces between these strong peaks correspond to the time when the call connection is not established.

Figure 5 shows spectrum of DTV signal. In Figure 7, a strong peak around $700 \mathrm{MHz}$ frequency corresponds to the pilot signal of the sensed DTV channel. The frequency span is $5 \mathrm{MHz}$ and this signal is broadcasted by the local television station and the transmitter is in line of sight (LOS) from the sensing location with the aerial distance of around 10 miles [9] [11].

Table 1 shows testing of each channel for only $30 \mathrm{msec}$ considering the idle and busy time observations. Further on the basis of these observations cognitive radio could take the decisions for the handoff mechanism [12] which will minimize the time required for the handoff mechanism. Especially service time for deciding which channel is less in our paper. Further the information from the Table 1 is used as a database visitor list for finding the secondary user while taking the decision of spectrum handoff mechanism.

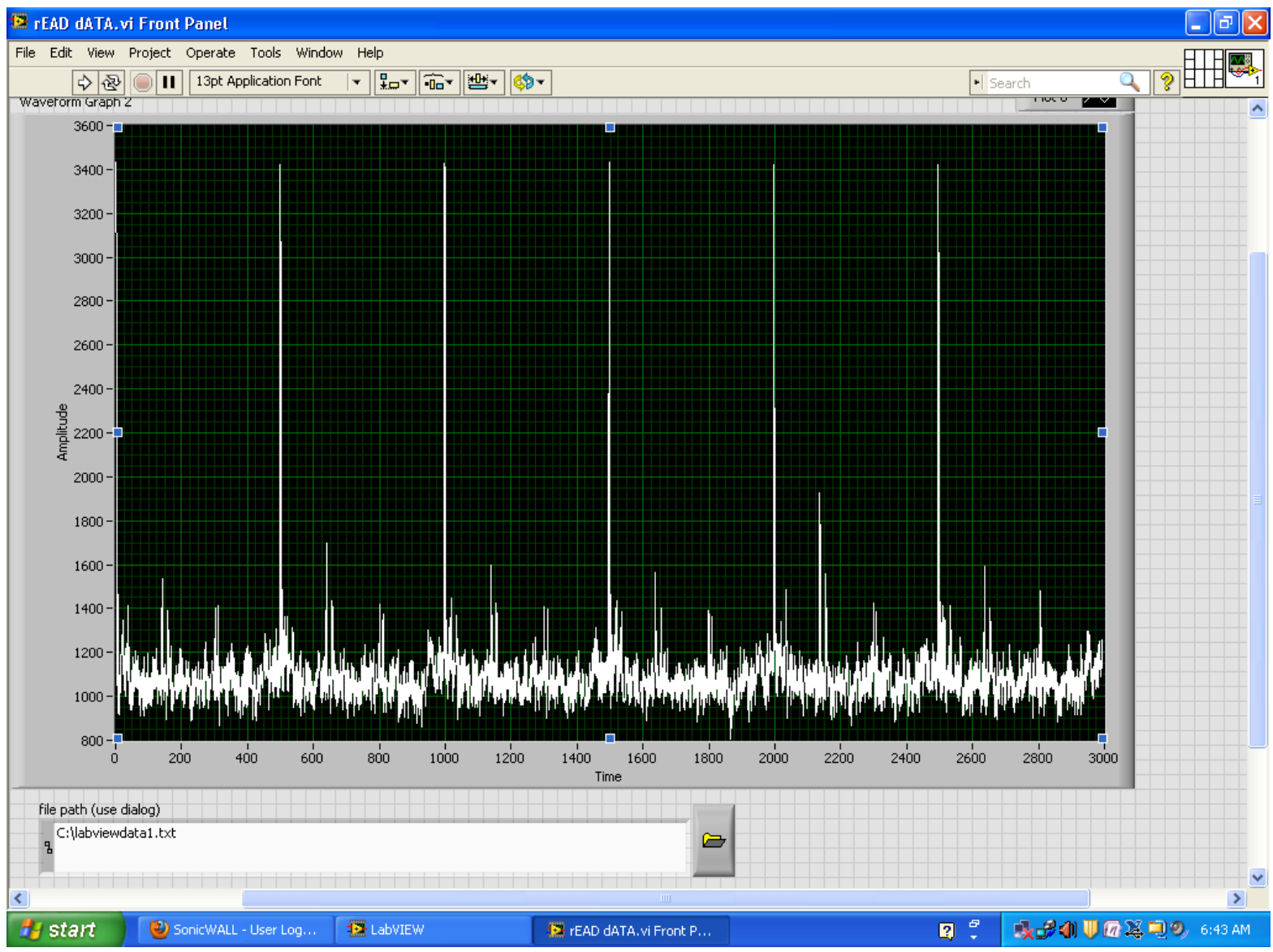

Figure 3. Spectrum 1 (CDMA). 


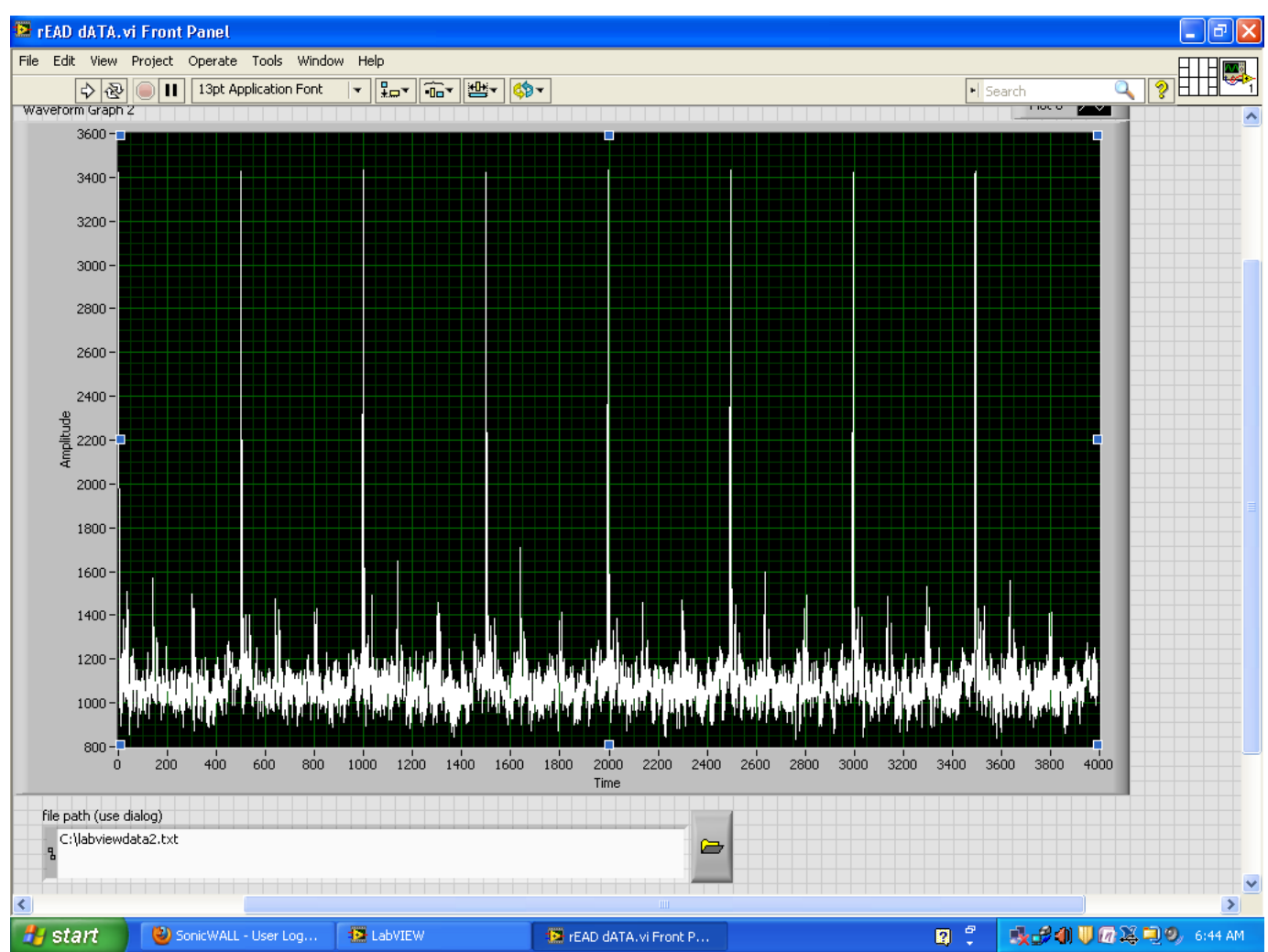

Figure 4. Spectrum 2 (GSM).

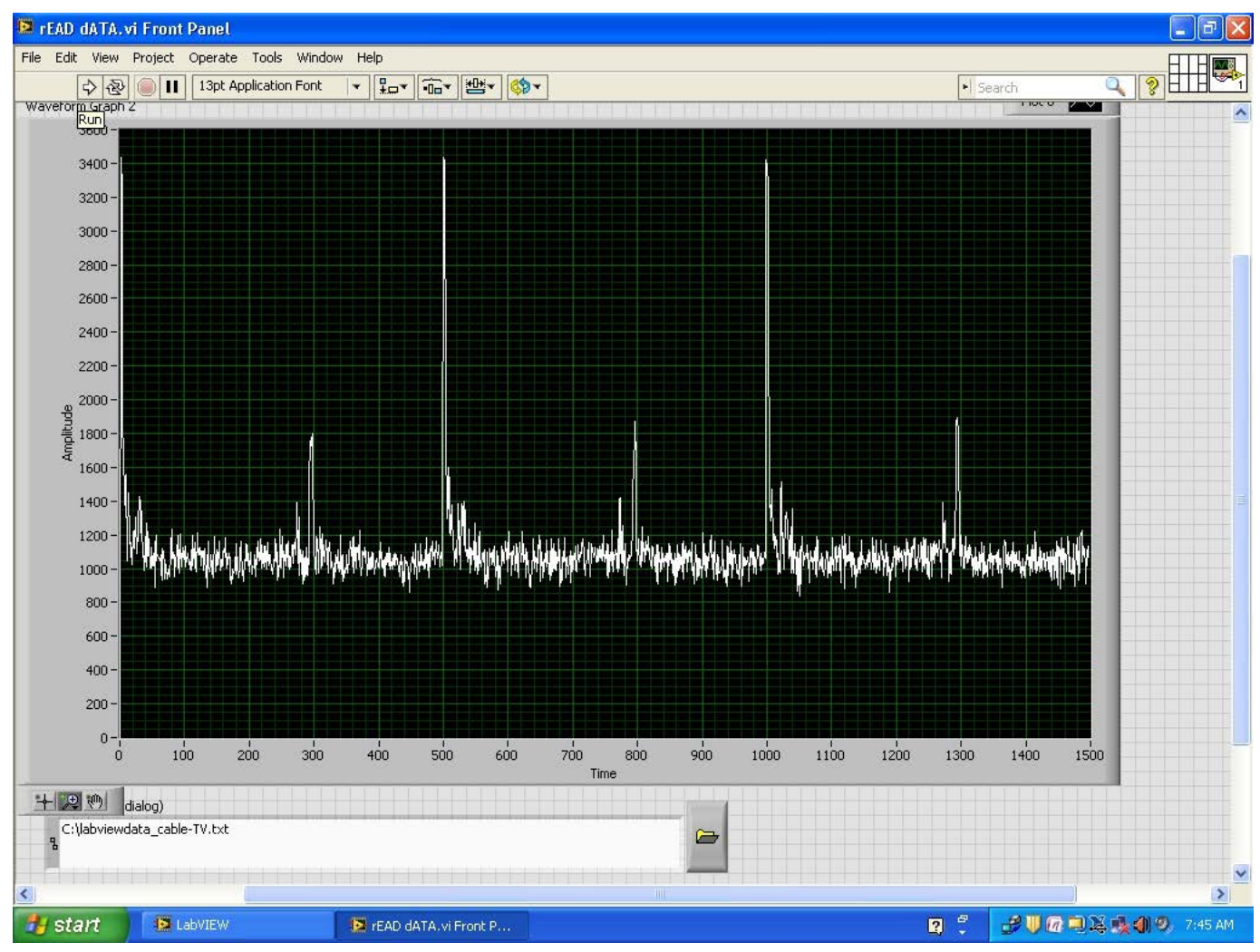

Figure 5. Spectrum 3 (DTV). 
Table 1. Database for cognitive radio.

\begin{tabular}{cccc}
\hline Type of spectrum & Busy time (msec) & Idle time (msec) & Channel used \\
\hline Spectrum 1 & 20 & 10 & 1 \\
Spectrum 2 & 25 & 5 & 2 \\
Spectrum 3 & 20 & 10 & 3 \\
Spectrum 4 & 15 & 15 & 4 \\
\hline
\end{tabular}

\section{Spectrum Handoff}

Before a data transmission starts, SUs hop through the channels following the same hopping sequence. Once a successful RTS/CTS handshake between SU transmitter and its receiver takes place. Consider the system which consists of two types of 20 radio users, primary users and secondary users i.e. cognitive radios operating in the same spectrum band with SU traffic fixed at $s=1$ [13] [14]. The spectrum band consists of $M$ primary bands and each band is divided in $N$ sub-bands. In our model greedy channel selection scheme is always preferred for the selection of channel with minimum service time. The probability of collision which occurs among SUs needs to be considered for performing spectrum handoff by the more than one SUs at the same time [15] [16]. Thus the probability that a collision occurs among SUs is given by.

Channel selection scheme, on the selected channel both the SU transmitter and receiver do not need to exchange information. When multiple SUs perform spectrum handoff at the same time, a pseudo random selecting sequence for each $\mathrm{SU}$ is generated locally.

To avoid collision same selecting sequence is used for the selection of channel. In our model, data base with the information of free channels is always with the SUs. Hence the channel selection time and collision which is the trouble in [15] [16] is reduced in our proposed spectrum handoff scheme. Cognitive radio calls to idle-subbands based on the database with it. Based on that reallocation of band, spectrum handoff can be performed by the base station centrally.

\subsection{Optimal Spectrum Handoff}

In the previous PU activity prediction schemes such as [17] [18], the authors propose the spectrum switching algorithm based on the channel idle probability sequence. When PU signal is detected, the channel with highest probability of being idle at next time slot is chosen to be the first candidate channel. This approach has been proven that can effectively reduce the spectrum switching delay. However, it does not consider the potential available transmission time of the channel. The channels with high idle probability may have considerable short available time, which causes inevitable frequent channel switches of secondary communication. Spectrum switch process includes time for the spectrum decision process, signaling for establishing new channels, and RF front-end reconfiguration. Hence, CR networks need to keep the handoff times as low as possible. We propose an optimal spectrum handoff scheme considers not only the probability of being idle, but also the potential idle durations and the expected transmission time. Once PU signal is detected and confirmed through fast and fine sensing stage, with the assistance of nearby users, CR can get the previous sensing cycle's spectrum occupancy status of the rest $N-1$ channels. Then the CR user can predict the probability of candidate channel availability at the current handoff moment by utilizing the most recent sensing results [19].

\subsection{Geographical Handoff}

For the fast handoff procedure geographical handoff procedure is developed. Our system provides primary users (PUs) functionality to the secondary user which is connected first as long as the user roams inside a specific range of SUs. This range is found through a global angle $(\theta)$ and the distance between the first SU and PU [20] [21] is $d_{1}$.

Suppose $R$ is distance from the global $\theta$ angle calculated as follows:

$$
R=d_{1} * \tan (q)
$$

where the distance between previous SU and PU, $d_{1}$; 
Suppose the distance between new SU and PU, $d_{2}$.

The procedure of changing domains without inter-domain fast handoffs upon reception of the registration request from a main station is as follows:

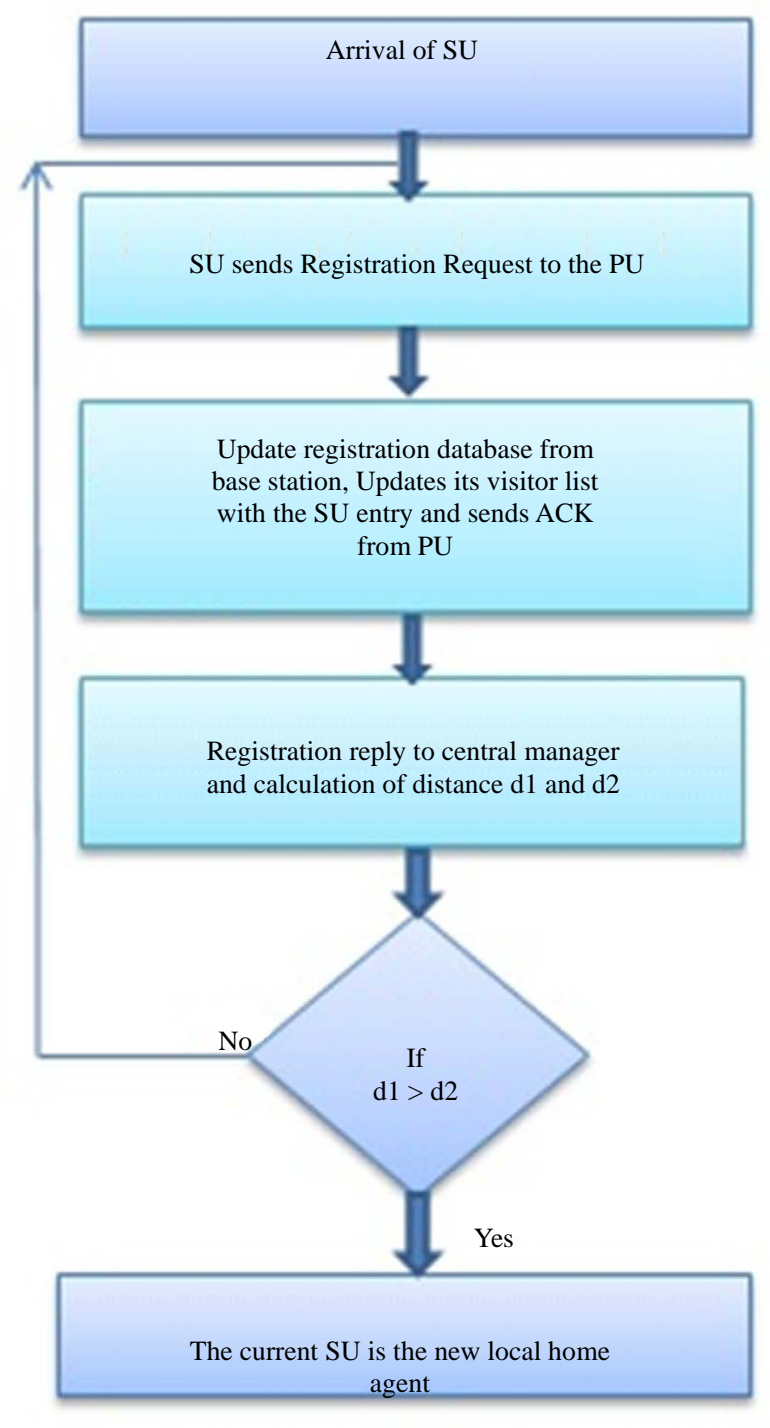

Flowchart for Geographical Handoff Mechanism

\subsection{Proposed Spectrum Handoff Scheme}

In case of spectrum handoff schemes used in A and B central identity i.e. manager is required for taking decision of spectrum handoff whereas in our proposed scheme we used distributed handoff is used for channel coordination. The received signal $r(t)$ is applied to the energy detector with bi-thresholds which are used for detection of primary user. If the received energy is less than threshold then the PU is absent and if the received energy is greater than threshold then the presence of PU is detected. If the energy detected is in between two threshold values then $\mathrm{U}$ is considered in the region of uncertainty. The energy spent for the detection of primary user (PU) is calculated as follows.

An energy detector with bi-thresholds is used for detection in which two thresholds $\mathrm{H}_{0}$ and $\mathrm{H}_{1}$ are used. Consider $r(t)$ is the received signal, $n(t)$ is the additive white Gaussian noise (AWGN) and $h$ is the amplitude of the channel. The received energy is given by 


$$
r(t)= \begin{cases}n(t) & \text { if } \mathrm{H}_{0} \\ n(t)+h s(t) & \text { if } \mathrm{H}_{1}\end{cases}
$$

where $\mathrm{H}_{0}$ indicates only noise and $\mathrm{H}_{1}$ indicates presence of PU.

Multiple PU systems are considered for detection. It is assumed that, for some PU systems, the signal structure is unknown, while for the others, enough information is known about the PU waveform to perform the energy detector as an optimal detector. It is assumed that there are $N$ channels to be sensed. The SU will scan the whole spectrum and detect whether or not there is a spectrum hole available. The SU identifies that a PU waveform is known (or not) on the basis of the power and band of interest and this information using AODV routing protocol is further used in terms of threshold for the detection of spectrum hole the packet broadcasting and handoff mechanism.

The overall probability of detection and the probability of false alarm are given as

$$
\begin{aligned}
& P_{d}=P_{r} P_{d, E C} \\
& P_{f}=P_{r} P_{f, E C}
\end{aligned}
$$

For maximum channels assume $0.5<P_{r} \leq 1$. The SU will perform energy detector for sensing the majority of channels. By assuming $P_{r} \approx 1$, the probability of detection, the probability of false alarm, and the mean detection time are calculated.

The output $E$ of the energy detector has a distribution that is defined as follows [13].

$$
E= \begin{cases}X_{2 W}^{2} & \text { If } \mathrm{H}_{0} \\ X_{2 W}^{2}(2 \gamma) & \text { If } \mathrm{H}_{1}\end{cases}
$$

where $X_{2 W}^{2}$ and $X_{2 W}^{2}(2 \gamma)$ represent a central chi-square distribution and a non-central chi-square distribution with $2 w$ degrees of freedom and the non-centrality parameter $2 \gamma$, respectively. The approximation for the probability of detection $P_{d, E}$ and probability of false alarm $P_{f, E}$ of the energy detector.

In the previous PU activity prediction schemes such as [17] [18], the authors propose the spectrum switching algorithm based on the channel idle probability sequence. When PU signal is detected, the channel with highest probability of being idle at next time slot is chosen to be the first candidate channel. This approach has been proven that can effectively reduce the spectrum switching delay. However, it does not consider the potential available transmission time of the channel. The channels with high idle probability may have considerable short available time, which causes inevitable frequent channel switches of secondary communication. Spectrum switch process includes time for the spectrum decision process, signaling for establishing new channels, and RF front-end reconfiguration. Hence, CR networks need to keep the handoff times as low as possible. We propose an optimal spectrum handoff scheme considers not only the probability of being idle, but also the potential idle durations and the expected transmission time. Once PU signal is detected and confirmed through fast and fine sensing stage, with the assistance of nearby users, CR can get the previous sensing cycle's spectrum occupancy status of the rest $N-1$ channels. Then the CR user can predict the probability of candidate channel availability at the current handoff moment by utilizing the most recent sensing results [19].

\section{Performance Evaluation}

In order to evaluate the performance of the proposed handoff mechanism and comparison of the proposed handoff mechanism with the optimal handoff mechanism, we implement a network simulator to support network topology consisting of multiple cells. Here base stations are assumed to update after every 1 hour availability of free spectrum to cognitive radio so that there will be less service time required for the secondary users after arrival of primary user for the data transmission. In this simulation we investigate throughput and average delay using optimal handoff mechanism and geographic handoff mechanism. We compare the proposed method with the optimal spectrum handoff as shown in Figure 6 and Figure 7 respectively. Transreceiver section required for the paging is always with the cognitive radio which moves within the cell and continuously updates the data base which is with the base station. Each CR equipped with one transceiver moves into the cell and periodically sense and transmits on one spectrum band, which is selected by following the spectrum decision rule. For the 


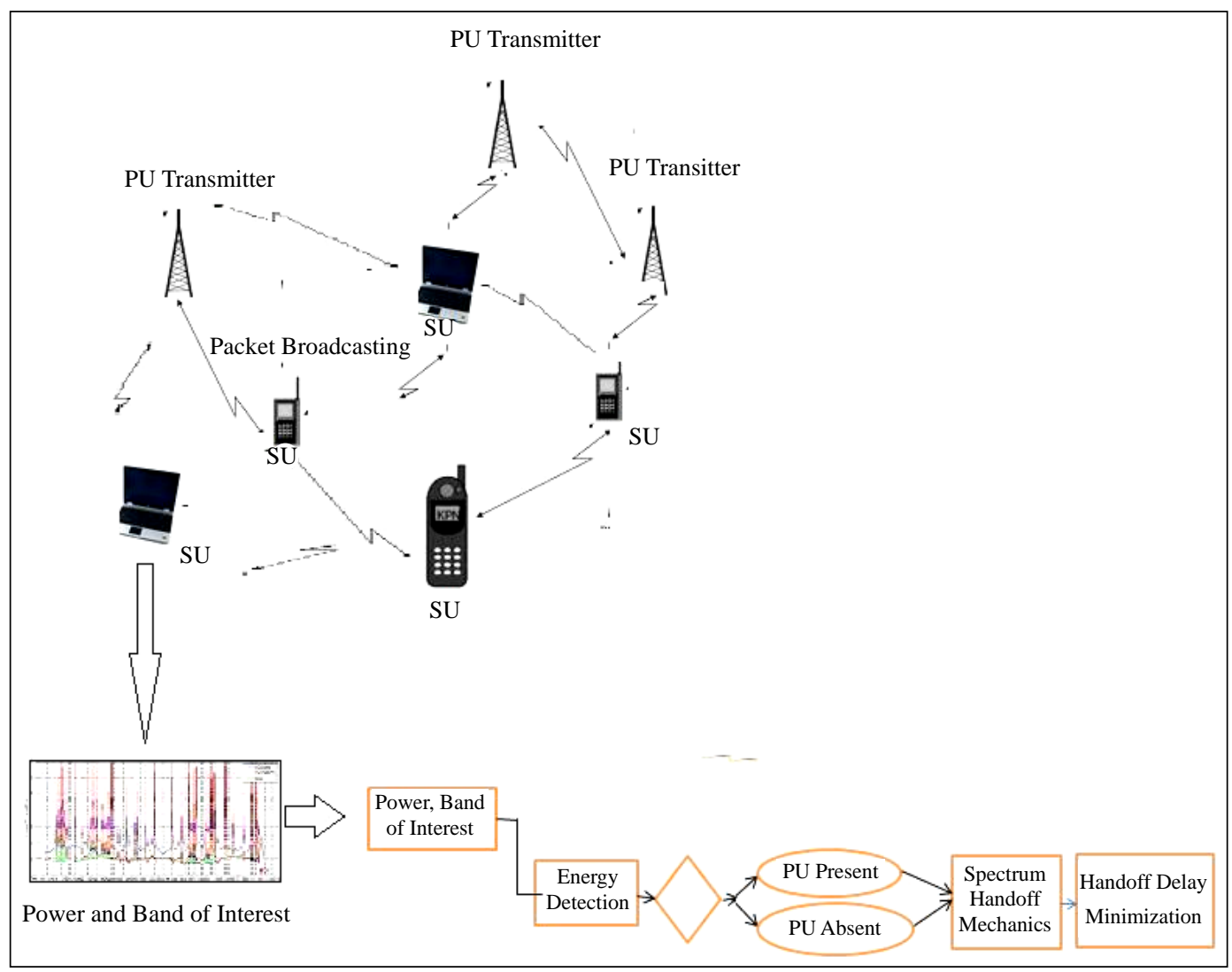

\section{Figure 6. System model.}

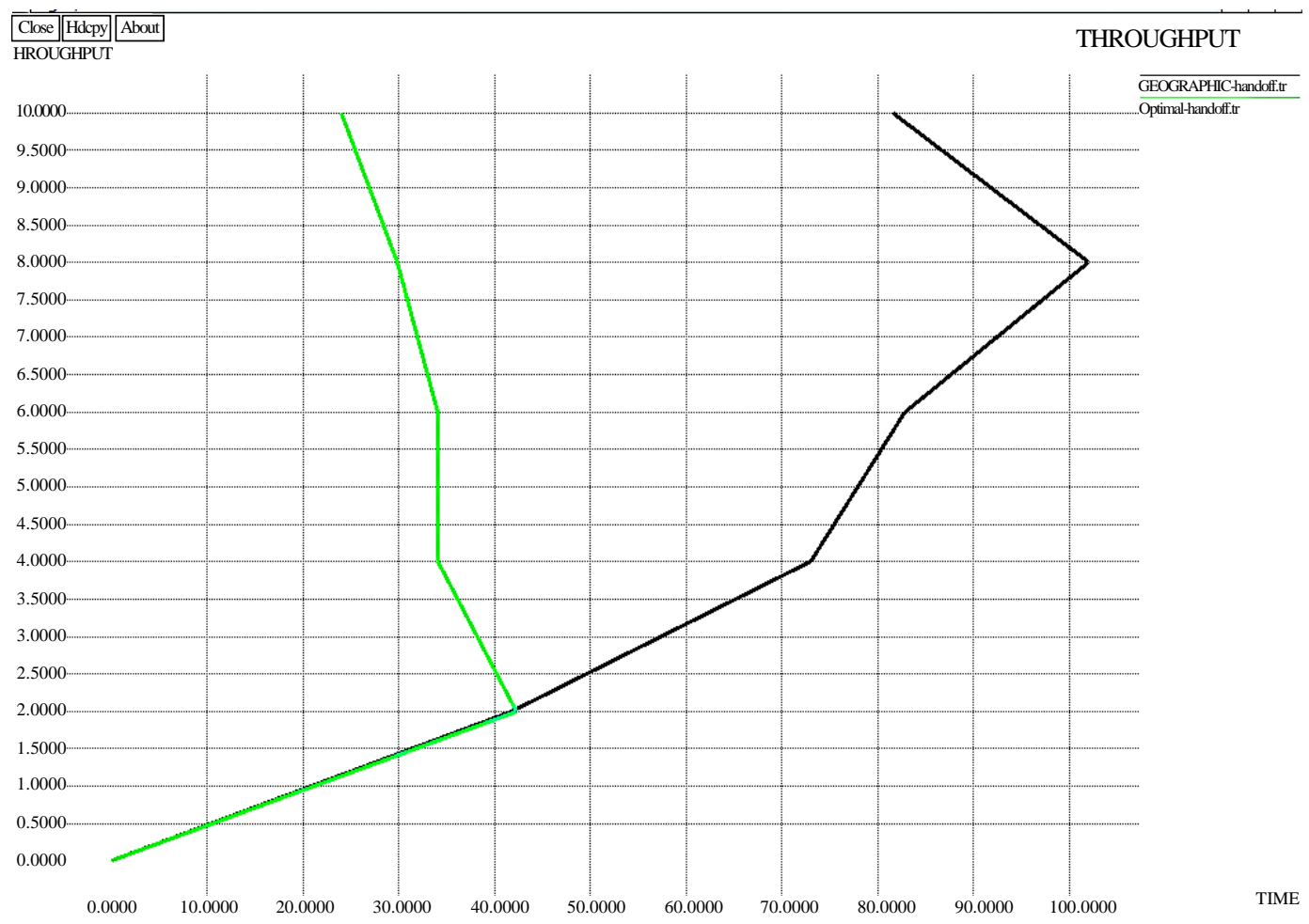

Figure 7. Transmission time with/without cooperation. 
selection of channel energy detection technique is applied and the average signal-to-noise ratio (SNR) is assumed to be known to all CRs. We further assume CRs in the same cell can exchange information through dedicated common control channel (CCC) is used [22]. Figure 7 shows the numbers of spectrum handoff times under two different handoff schemes when CR transmits 10 - $100 \mathrm{Mb}$ data.

We verify the effects of handoff cooperation and show the results in Figure 8. We observe that the average spectrum handoff delay is target channel status in advance with the assistance of nearby users. We observe the proposed handoff scheme has the least handoff times comparing to optimal handoff schemes since it can systematically consider the influence of potential channel availability and the expected transmission time. It proves that our proposed approach has the improved efficient performance on reducing CR system spectrum handoff delay.

For the study of space domain spectrum handoff, we let the CR user moves between different cells with velocity of $10 \mathrm{~m} / \mathrm{s}$ and $50 \mathrm{~m} / \mathrm{s}$, and measure the spectrum handoff times with or without space domain channel switching as shown in Figure 8. As the moving speed turns to be high which means CR passes more cells, we observe more spectrum handoffs occur without cell switching prediction. It validates that the proposed space domain handoff approach can efficiently reduce the handoff times.

\section{Conclusion}

This paper deals with spectrum detection and spectrum handoff mechanism in cognitive radio network. Spectrum analyzer is used to emulate cognitive radio to do spectrum sensing. The whole procedure of spectrum sensing including sensing setup, instrument control, sensing capability, sensing scenario and sensing result is presented in detail. The main advantage of equipment-based spectrum sensing is to perform quick and semicontinuous measurements. The time needed for each measurement is around 80 - 110 ms. Spectrums of CDMA signal, GSM signal and DTV signal are shown in this paper. Based on this information, the information about the spectrum is embedded in packets and broadcasted to every user. Moreover these spectrum measurements

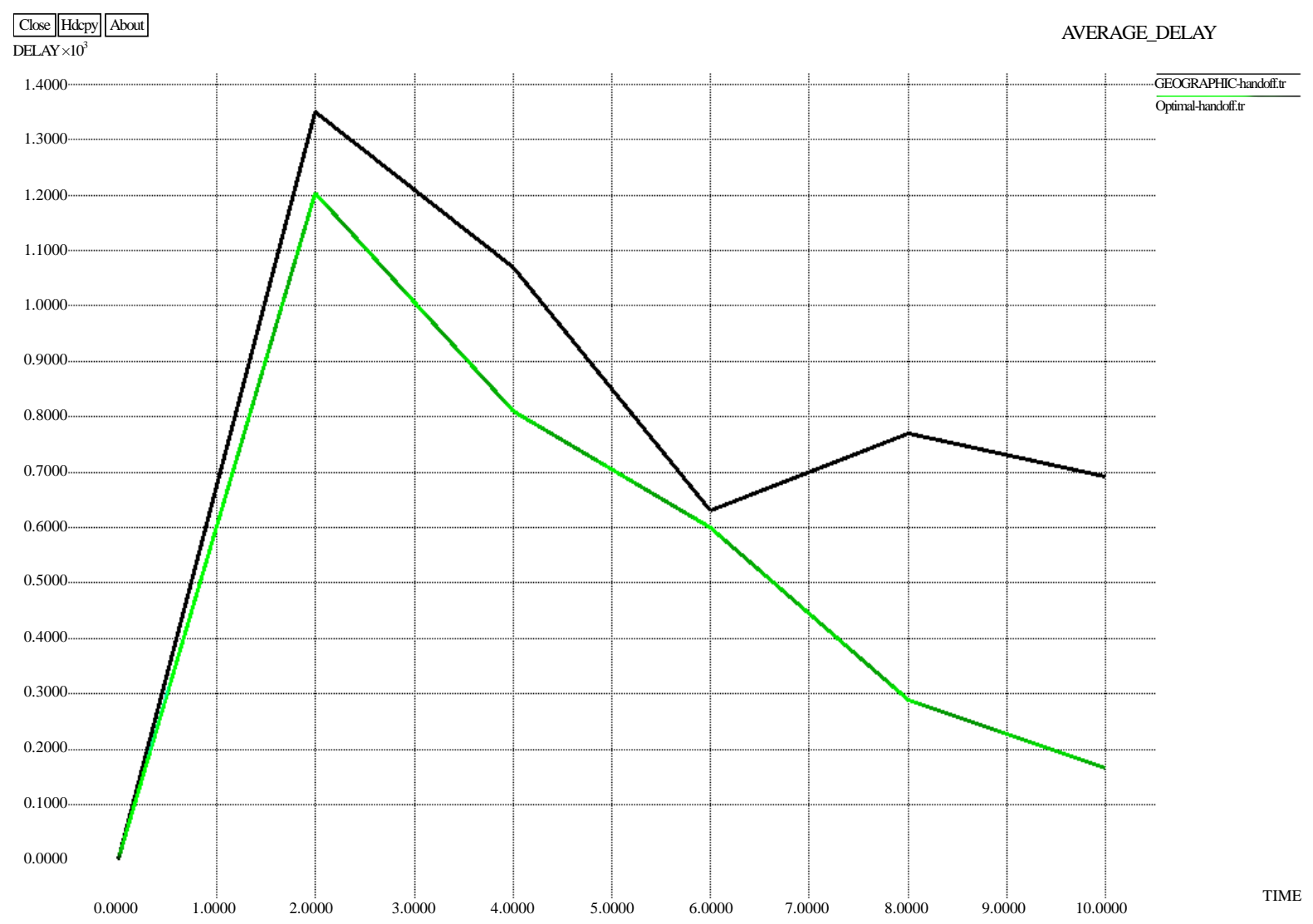

Figure 8. Handoff times with/without cooperation under different speed. 
used for taking the decision about spectrum handoff mechanism. The results are compared with the conventional spectrum sensing method and observed considerable improvement in the minimization of spectrum handoff time.

\section{Acknowledgements}

This work is funded by the BCUD section, university of Pune through a grant (11ENG000378). The authors wants to thank to parent institute MIT academy of Engineering and MITCOE, Pune and research institute S.V. National Institute of Technology, Surat, Gujrat for their valuable support for carrying this research.

\section{References}

[1] Mitola, J. (2005) Cognitive Radio: Making Software Radio More Personal. IEEE Personal Communications, August 1999.

[2] Federal Communications Commission (FCC) (2006) Notice of Proposed Rule Making. Et Docket No. 06-156, October.

[3] Cordeiro, C., Challapali, K., Birru, D. and Sai Shankar, N. (2006) IEEE 802.22: An Introduction to the First Wireless Standard Based on Cognitive Radios. Journal of Communications, 1, 38-47. http://dx.doi.org/10.4304/jcm.1.1.38-47

[4] Hossain, E. and Bhargava, V. (2007) Cognitive Wireless Communication Networks. Springer Science Business Media, Berlin. http://dx.doi.org/10.1007/978-0-387-68832-9

[5] Zhou, X.W., Ma, J., Li, G.Y., Kwon, Y.H. and Soong, A.C.K. (2009) Probability-Based Optimization of Inter-Sensing Duration and Power Control in Cognitive Radio. IEEE Transactions on Wireless Communications, 8, $4922-4927$. http://dx.doi.org/10.1109/TWC.2009.081061

[6] Quan, Z., Cui, S.G. and Sayed, A.H. (2008) Optimal Linear Cooperation for Spectrum Sensing in Cognitive Radio Networks. IEEE Journal of Selected Topics in Signal Processing, 2, 28-40. http://dx.doi.org/10.1109/JSTSP.2007.914882

[7] Ye, Z., Memik, G. and Grosspietsch, J. (2008) Energy Detection Using Estimated Noise Variance for Spectrum Sensing in Cognitive Radio Networks. IEEE Communications Society, WCNC 2008 Proceedings, Las Vegas, 31 March-3 April 2008, 711-716.

[8] Liang, Y.-C., Zeng, Y.H., Peh, E.C.Y. and Hoang, A.T. (2008) Sensing-Throughput Tradeoff for Cognitive Radio Networks. IEEE Transactions on Wireless Communications, 7, 1326-1337. http://dx.doi.org/10.1109/TWC.2008.060869

[9] Yucek, T. and Arslan, H. (2009) A Survey of Spectrum Sensing Algorithms for Cognitive Radio Applications. IEEE Communications Surveys \& Tutorials, 11, 116-130. http://dx.doi.org/10.1109/SURV.2009.090109

[10] Wang, H., Noh, G., Kim, D., Kim, S. and Hong, D. (2010) Advanced Sensing Techniques of Energy Detection in Cognitive Radios. Journal of Communications and Networks, 12, 19-29. http://dx.doi.org/10.1109/JCN.2010.6388431

[11] Kim, H. and Shin, K.G. (2008) In-Band Spectrum Sensing in Cognitive Radio Networks: Energy Detection or Feature Detection? Real-Time Computing Laboratory, EESC Department the University of Michigan, Ann Arbor.

[12] Chen, R.L., Park, J.M. and Bian, K.G. (2006) Robust Distributed Spectrum Sensing in Cognitive Radio Networks. Technical Report Tr-Ece-06-07, Department of Electrical and Computer Engineering.

[13] Haykin, S., Thomson, D.J. and Reed, J.H. (2009) Spectrum Sensing for Cognitive Radio. Proceedings of the IEEE, 97, 849-877. http://dx.doi.org/10.1109/JPROC.2009.2015711

[14] Saini, A.S., Hu, Z. and Qiu, R. (2009) Spectrum Sensing and Reconstruction for Cognitive Radio. Department of Electrical and Computer Engineering, Center for Manufacturing Research.

[15] Song, Y. and Xie, J. (2011) On the Spectrum Handoff for Cognitive Radio Ad-Hoc Network without Common Control Channel. Springer Science Business Media LLC, Berlin.

[16] Liang, Y.C., Zeng, Y.H., Peh, E.C.Y. and Hoang, A.T. (2008) Sensing Throughput Tradeoff for Cognitive Radio Networks. IEEE Transactions on Wireless Communication, 7, 1326-1337. http://dx.doi.org/10.1109/TWC.2008.060869

[17] Cesana, M., Cuomo, F. and Ekici, E. (2010) Routing in Cognitive Radio Networks: Challenges and Solutions. Ad Hoc Networks, 9, 228-248. http://dx.doi.org/10.1016/j.adhoc.2010.06.009

[18] Di Felice, M., Choudhury, K.R., Kim, W., Kassler, A. and Bononi, L. (2010) End-to-End Protocols for Cognitive Radio Ad-Hoc Networks: An Evaluation Study. Performance Evaluation, 68, 859-875. http://dx.doi.org/10.1016/j.peva.2010.11.005

[19] Lee, W.Y. and Akhilidiz, I.F. (2012) Spectrum Aware Mobility Management in Cognitive Radio Cellular Networks. IEEE Transactions on Mobile Computing, 11, 529-542. http://dx.doi.org/10.1109/TMC.2011.69 
[20] Balogun, V. (2010) Challenges of Spectrum Handoff in Cognitive Radio Networks. Pacific Journal of Science and Technology, 11, 304-314.

[21] Song, Y. and Xie, J. (2011) Performance Analysis of Spectrum Handoff for Cognitive Radio Ad-Hoc Networks without Common Control Channel under Homogeneous Primary Traffic. arXiv:1105.0031v3

[22] Duan, J.Q. and Li, Y. (2011) An Optimal Spectrum Handoff Scheme for Cognitive Radio Mobile Ad-Hoc Networks. Advances in Electrical and Computer Engineering, 11, 11-16. http://dx.doi.org/10.4316/aece.2011.03002 
Scientific Research Publishing (SCIRP) is one of the largest Open Access journal publishers. It is currently publishing more than 200 open access, online, peer-reviewed journals covering a wide range of academic disciplines. SCIRP serves the worldwide academic communities and contributes to the progress and application of science with its publication.

Other selected journals from SCIRP are listed as below. Submit your manuscript to us via either submit@scirp.org or Online Submission Portal.
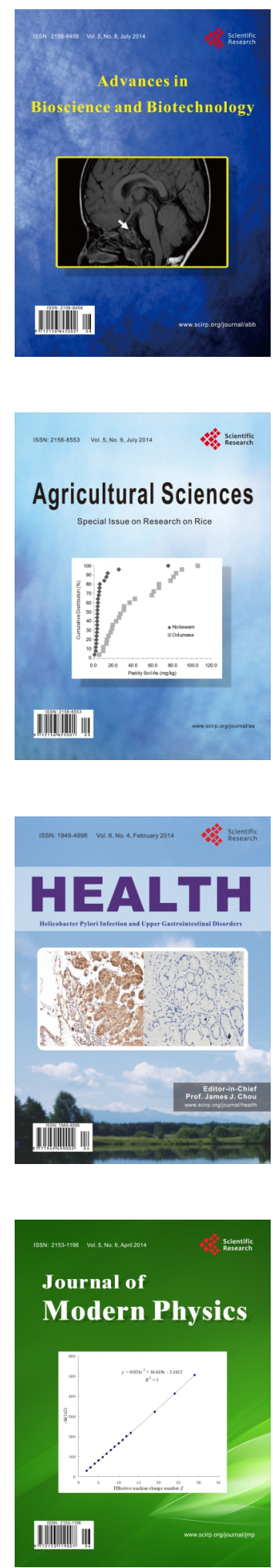
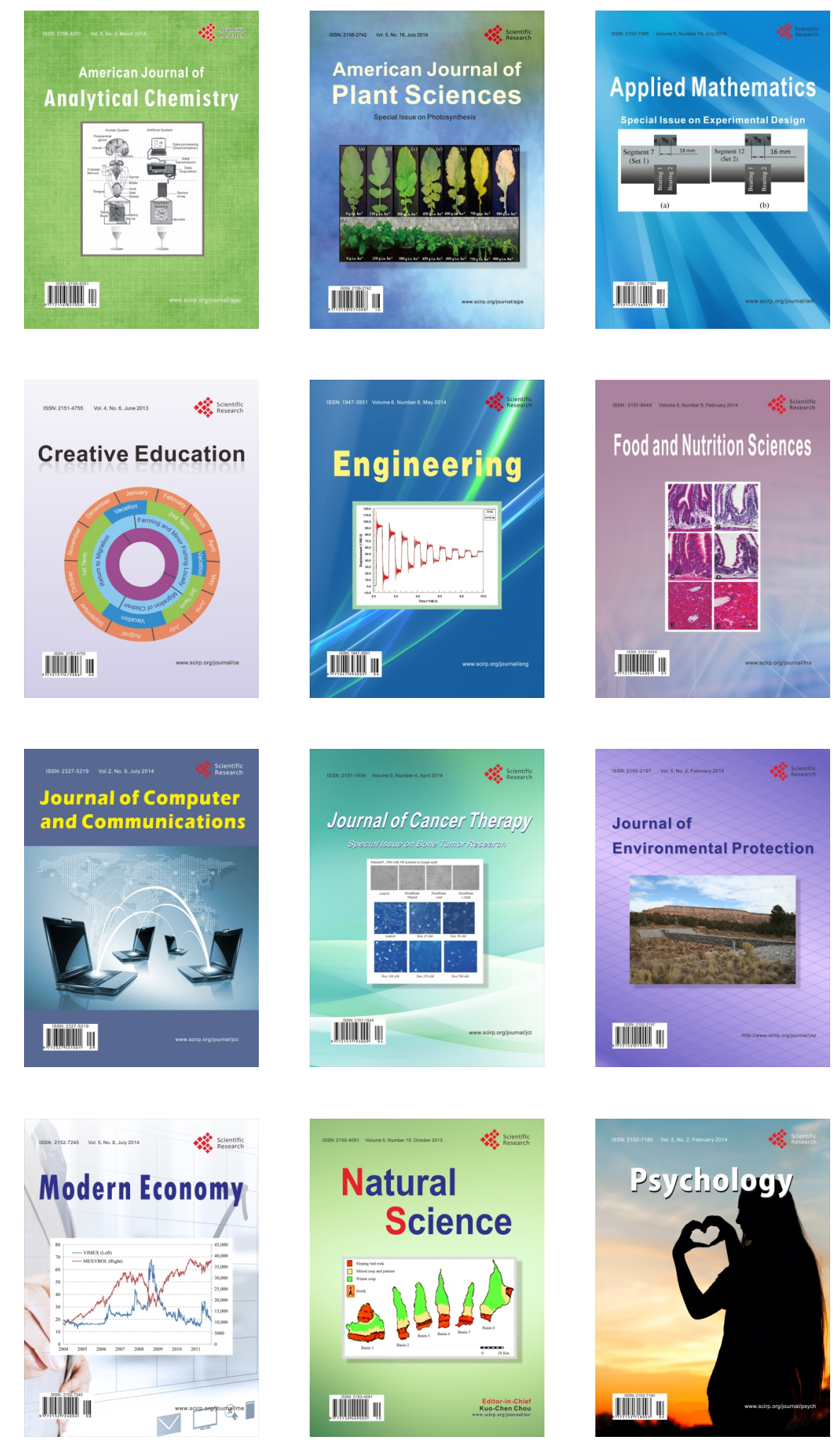